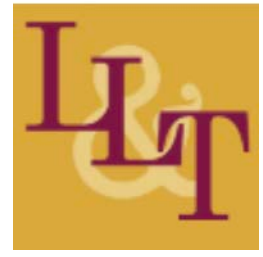

\title{
Announcements and Acknowledgements
}

APA Citation: Announcements and acknowledgements. (2018). Language Learning \& Technology, 22(1), i-ii. doi:10125/44590

\section{Announcements}

$L L T$ would like to announce our new website available at www.lltjournal.org.

We offer a sincere thank you to our sponsoring organizations, the National Foreign Language Resource Center (NFLRC) for creating the new website and the Center for Language Education and Research (CLEAR) for assisting with the transfer of the content.

We express our heartfelt thanks to Markus Kötter, Jill Pellettieri, and Phillip Towndrow for serving on our editorial board. Their service and support for our journal has been exemplary. In addition, we would like to welcome Jack Burston, Yu-Ju Lan, and Mimi Li to the board. We cannot wait to work with them in the future.

\section{LLT Reviewer Acknowledgements}

The editorial staff would like to recognize the reviewers of submitted $L L T$ manuscripts during 2017. This journal could not function without the academic service of these scholars, and we would like to thank them warmly for their contribution.

Lee Abraham, Zsuzsanna Abrams, Yuka Akiyama, Antonie Alm, Enza Antenos, Scott Aubrey, Richard Bale, Francis Bangou, Uwe Baumann, Phil Benson, Dawn Bikowski, Robert Blake, Alex Boulton, Dan Brown, Jack Burston, Mónica Cárdenas-Claros, Marinella Caruso, Catherine Caws, Luis Cerezo, Cheryl Wei-yu Chen, Hsin-I Chen, Rainbow Chen, Yan Chen, Alice Chik, Sunah Cho, Cathy Cohen, Joseph Collentine, Karina Collentine, Kathy Conklin, Grainne Conole, Troy Cox, Catia Cucchiarini, Sylvie De Cock, Jonathan deHaan, Tracey Derwing, Melinda Dooly, Stayc DuBravac, Laura Ducate, Richmond Dzekoe, Frieda Fichtner, Carlton Fong, Ana Frankenberg-Garcia, Mark Freiermuth, Carolin Fuchs, Rod Gardner, Nazlınur Göktürk, Sean Grant, Paul Gruba, Gabriel Guillen, Christoph Hafner, Marie-Josee Hamel, Sumi Han, Debra Hardison, Mohammad Hassanzadeh, Mirjam Hauck, Francesca Helm, Alan Hirvela, Hsiu-Chen Hsu, Hui Huang, Daniel Isbell, Sake Jager, Min Jung Jee, Qian Kan, Nenagh Kemp, Claire Kennedy, Richard Kern, Greg Kessler, Daesang Kim, Michelle Knobel, Claudia Kost, Markus Kötter, Dennis Koyama, Jeff Kuhn, Derya Kulavuz-Onal, Malgorzata Kurek, Kristopher Kyle, Geoff LaFlair, Chun Lai, Eva Lam, Yvonne Lam, Yu-Ju Lan, Joshua Lawrence, Sabine Levet, Jinrong Li, Mimi Li, Meei-Ling Liaw, Anthony Liddicoat, Chin-Hsi Lin, Dilin Liu, Paul Lyddon, David Malinowski, Michael Marek, Rachel Martin, Antonio Carlos Martins, Adam Mendelson, Sarah Mercer, Hui-Tzu Min, Katia Monteiro, Maribel Montero Perez, Liam Murray, Phuong Nguyen, Elke Nissen, Breffni O’Rourke, Ana Oskoz, Ana Padial, Luisa Panichi, Magali Paquot, Youngmin Park, Mark Pegrum, Carmen Perez-Llantada, Lucy Pickering, Arja Piirainen-Marsh, Anthippi Potolia, Hayo Reinders, Jonathon Reinhardt, Anne Rimrott, Tom Robb, Amy Rossomondo, Susanne Rott, Jack Rouzer, Kyle Scholz, Jean Schultz, Mack Shelley, Gillian Skyrme, Simon Smith, Susana Sotillo, Ursula Stickler, Glenn Stockwell, Tetyana Sydorenko, Denyse Toffoli, Constanza Tolosa, Ekaterina Tour, Chen-Hui Tsai, Anna Turula, Robert Vanderplank, Erik Voss, 
Ute Walker, Shudong Wang, Yanlin Wang, Paige Ware, Mark Warschauer, Cynthia White, Masanori Yamada, Chunsheng Yang, Jie Chi Yang, Iñígo Yanguas, Peng Yin, Choongil Yoon, Lihua Zhang, Binbin Zheng.

\section{LLT Copy Editor Acknowledgements for Volume 22, Issue 1}

The editorial staff would like to thank the copy editors that edited the manuscripts appearing in this issue. We appreciate their dedication and service while helping to prepare these manuscripts for publication.

Matt Bird (Brigham Young University), Tahnee Bucher Barbosa da Silva (The University of Arizona), Jennifer Gray (University of Wisconsin - Madison), Bronson Hui (Michigan State University), Nicolas Pares (University of Denver), Clemence Richard (Portland State University), Kristin Rock (The University of Hawai'i at Mānoa), Paul Spijkerbosch (J. F. Oberlin University). 